\title{
High-Performance Computing Course Development for Cultivating the Generalized System-level Comprehensive Capability
}

\author{
Juan Chen \\ College of Computer \\ National University of Defense Technology \\ Changsha, Hunan Province, China \\ juanchen@nudt.edu.cn
}

\begin{abstract}
Supercomputers are moving towards exascale computing [1], highperformance computer systems are becoming larger and larger, and the scale and complexity of high-performance computing (HPC) [2] applications are also increasing rapidly, which puts forward high requirements for cultivation of HPC majors and HPC course development [3]. HPC majors are required to be able to solve practical problems in a specific field of high-performance computing, which may be a problem for system design or a problem for a specific HPC application field. Regardless of the type of problem, the complexity and difficulty of the problem are often very high because HPC is interdisciplinary. The development of HPC courses to meet these kinds of talent cultivation needs must emphasize the cultivation of students' Generalized System-level Comprehensive Capabilities, so that students can master the key elements in the limited course knowledge learning process.

System-level Comprehensive Capability refers to the ability to use the knowledge and ability of the computer system to solve practical problems. The ACM/IEEE Joint Computer Science Curricula 2013 (CS2013) [4] also involves System-level Perspective. Systemlevel Comprehensive Capability is considered to be a crucial factor to improve students' system development ability and professional ability. This is especially important for students majoring in highperformance computing. Furthermore, due to the HPC field's interdisciplinary and high complexity characteristics, System-level Comprehensive Capability is not enough for HPC majors, and students need to have Generalized System-level Comprehensive Capabilities. A knowledge system at the computer system level "vertically" (from bottom to top: parallel computer architecture, operating system/resource management system, compilation, library optimization, etc.) is no longer enough; multiple high-performance computing application areas should also be "horizontally" involved. Generalized System-level Comprehensive Capabilities vertically and horizontally can meet the needs of different types of high-performance computing talents.
\end{abstract}

Permission to make digital or hard copies of all or part of this work for personal or classroom use is granted without fee provided that copies are not made or distributed for profit or commercial advantage and that copies bear this notice and the full citation on the first page. To copy otherwise, or republish, to post on servers or to redistribute to lists, requires prior specific permission and/or a fee. Copyright $\odot$ JOCSE, a supported publication of the Shodor Education Foundation Inc.

(c) 2021 Journal of Computational Science Education https://doi.org/10.22369/issn.2153-4136/12/2/7
How to cultivate the Generalized System-level Comprehensive Capabilities of HPC majors? National University of Defense Technology (NUDT) of China has faced some challenges [5] in building a series of high-performance computing courses, adopted some measures, and gained some experience [5-7]. NUDT has developed the Tianhe-2 supercomputer, which ranked No. 1 in the TOP500 list ${ }^{1}$ six times from June 2013 to November 2015. These achievements are inseparable from the training of high-performance computing talents and HPC curriculum development. NUDT has offered a series of high-performance computing courses from freshman to postgraduate for a long time. The courses cover a wide range, are difficult and practical, and pay great attention to the cultivation of students' Generalized System-level Comprehensive Capabilities. The following main means are adopted: i) Hierarchical capability model construction is used to guide the establishment of curriculum system and curriculum setting; ii) Real practice platforms and real cases from frontier scientific challenges are used to construct step-by-step practice cases; iii) A teaching mechanism that integrates scientific research and teaching content is adopted. In the curriculum setting, the emphasis is placed on basic mathematics, general science courses, high-performance computing professional courses, and basic courses for specific HPC application fields. Regarding the content of the curriculum, it is based on the principle of breaking the boundaries of disciplines and specialties, establishing the relevance of the knowledge system and frontier scientific issues, and designing the whole process of teaching content with the direct facing of basic scientific issues and frontier scientific research issues. In terms of course implementation methods, there are various forms, including small-class teaching, seminar-based teaching, casebased teaching, and flipped classrooms, etc. In the past ten years, the curriculum construction at NUDT has achieved remarkable results. We have cultivated Generalized System-level Comprehensive Capabilities of high-performance computing majors very well and greatly helped the development of our high-performance computing research.

\section{KEYWORDS}

High-performance computing, High-performance computing curricula, Generalized system-level comprehensive capability

$\overline{{ }^{1} \text { www.top500.org. }}$ 


\section{ACKNOWLEDGMENTS}

This work is supported by the 2019 Hunan Province Higher Education Teaching Reform Research Foundation of China (titled with "Teaching Practice of Training High-Performance Computing Talents Relying on High-level Scientific Research"), and the 2019 Hunan Province Postgraduate Outstanding Professional Case Foundation of China (titled with "High-Performance Computing Series Case Library").

\section{REFERENCES}

[1] Daniel A Reed and Jack Dongarra. Exascale computing and big data. Communications of the ACM, 58(7):56-68, 2015.

[2] NetApp, Inc. What Is High-Performance Computing?, 2019. https://www.netapp. com/us/info/what-is-high-performance-computing.aspx.

[3] Rajendra K. Raj, Carol J. Romanowski, John Impagliazzo, Sherif G. Aly, Brett A. Becker, Juan Chen, Sheikh Ghafoor, Nasser Giacaman, Steven I. Gordon, Cruz
Izu, Shahram Rahimi, Michael P. Robson, and Neena Thota. High performance computing education: Current challenges and future directions. In ITiCSE-WGR'20: Proceedings of the Working Group Reports on Innovation and Technology in Computer Science Education, pages 51-74, New York, NY, USA, 2020. ACM.

[4] Joint task force on computing curricula, association for computing machinery $(\mathrm{acm})$ and ieee computer society. computer science curricula 2013: Curriculum guidelines for undergraduate degree programs in computer science. Technical report, New York, NY, USA, 2013. 999133.

[5] Juan Chen, John Impagliazzo, and Li Shen. High-performance computing and engineering educational development and practice. In Proceedings of the 50th Frontiers in Education 2020 (FIE2020), pages 1-8, Uppsala, Sweden, 2020. IEEE

[6] Juan Chen, Li Shen, Jianping Yin, and Chunyuan Zhang. Design of practical experiences to improve student understanding of efficiency and scalability issues in high performance computing (poster). In Proceedings of the 49th ACM Technical Symposium on Computer Science Education (SIGCSE2018), pages 1090-1090, New York, NY, USA, 2018. ACM.

[7] Juan Chen, Yingjun Cao, linlin Du, Youwen Ouyang, and Li Shen. Improve student performance using moderated two-stage projects. In Proceedings of ACM Global Computing Education Conference (CompEd2019), pages 201-207, New York, NY, USA, 2019. ACM. 